\title{
Jian KANG \\ From dBA to soundscape indices: Managing our sound environment
}

(C) The Author(s) 2017. Published by Higher Education Press. This is an open access article under the CC BY license (http:// creativecommons.org/licenses/by/4.0)

\begin{abstract}
While in the EU alone 80 million citizens are suffering from excessive environmental noise, the conventional approach, i.e., reduction of 'sound level', does not always deliver the required improvements in quality of life. The growing field of 'soundscape studies' is addressing this gap by considering the sound environment as perceived, in context, with an interdisciplinary approach. However, soundscapes are hugely complex, and measuring them as a basis for environmental design requires a step change to the discipline. This paper explores the need for developing 'soundscape indices', in the movement from noise control to soundscape creation, adequately reflecting levels of human comfort, the impact of which will be reminiscent of that of the Decibel scale created by Bell Systems a century ago. By analysing the soundscape design of urban open public spaces, the coherent steps for achieving this are also discussed, including characterising soundscapes by capturing soundscapes and establishing a comprehensive database; determining key factors and their influence on soundscape quality based on the database; developing, testing and validating soundscape indices; and demonstrating the applicability of the soundscape indices in the management of our sound environment.
\end{abstract}

Keywords noise, soundscape, management, sound, environment, soundscape index

\section{Introduction}

The EU Green Paper on Future Noise Policy indicates that 80 million EU citizens are suffering from unacceptable

Received April 22, 2017; accepted May 15, 2017

Jian KANG (

School of Architecture, Harbin Institute of Technology, Harbin 150001, China

E-mail: j.kang@hit.edu.cn environmental noise levels according to the World Health Organization (WHO) recommendation (Berglund et al., 1999), and the social cost of transport noise is $0.2 \%-2 \%$ of total GDP. The conventional approach, namely reducing 'sound level', does not always deliver the required improvements in quality of life. The soundscape strategy, by considering the sound environment as perceived, in context, with an interdisciplinary approach, is a growing field for addressing this gap. However, soundscapes are rather complex and measuring them as a basis for environmental design requires a step change to the discipline.

This paper explores the need for developing 'soundscape indices' (SSID), adequately reflecting levels of human comfort. The paper first reviews the movement from noise control to soundscape creation; it then addresses the needs of changing from dBA-type of measures to soundscape indices. By analysing the soundscape design in urban open public spaces, the paper then proposes a framework for the development and application of soundscape indices. Finally, the significance of overall soundscape approach associated with soundscape indices is discussed.

\section{From noise control to soundscape crea- tion}

Environmental noise, including road/rail/air, industries, construction, public work and the neighbourhood, is often the main cause of environmental distress in terms of the number of complaints received. This is also a global problem, especially in developing countries, due to the rapid urbanisation and development of infrastructure. Potential noise effects include hearing impairment, startle and defence reactions, aural pain, ear discomfort, speech interference, sleep disturbance, cardiovascular effects, performance reduction, and annoyance. These health effects, in turn, can lead to social handicap, reduced 
productivity, decreased performance in learning, absenteeism in the workplace and school, increased drug use, and accidents (Berglund et al., 1999; Wilhelmsson, 2010). Noise also has economic impacts such as loss of property value.

The EU Directive Relating to the Assessment and Management of Environmental Noise (END) published in 2002 has led to a number of major actions. Reducing noise level has been the focus in the Directive, as well as in all the other current regulations and policies worldwide, with billions of euros are being spent. However, reducing sound level is not always feasible and cost-effective, and more importantly, will not necessarily lead to improved quality of life, although considerable research and practical work have been carried out in noise abatement in the last several decades. For example, previous studies in urban open spaces have shown that when the sound level is below a certain value (as high as 65-70 dBA), people's acoustic comfort evaluation is not related to the sound level, whereas the sound type, the user characteristics, and other factors play an important role (Kang, 2007). Moreover, the importance of considering the overall sound environment rather than just noise sources (especially road traffic) may also become more significant with the development of quieter vehicles, a major action along with the EU Directive. Furthermore, it has been shown that the environmental noise annoyance only depends on approximately $30 \%$ of the physical facets of parameters such as acoustic energy (Guski, 1997). The END has also called for action on "quiet areas"-A particular type of soundscape that is worth preserving. Some "quiet area mapping' is being explored, but it is not clear how to determine those areas, where to go with it, or how to use it, or how to incorporate it in design. Therefore, a step change is needed, by developing a new method to assess sound environment quality.

Soundscape creation, different from noise control engineering, is about relationships between the ear, human beings, sound environments, and society. It is related to many disciplines including acoustics, aesthetics, anthropology, architecture, ecology, ethnology, communication, design, human geography, information, landscape, law, linguistics, literature, media arts, medicine, musicology, noise control engineering, philosophy, pedagogics, psychology, political science, religious studies, sociology, technology and urban planning. Soundscape also has significant practical relevance in terms of policies as well as the planning/design process. Although the term soundscape was introduced in the 1960s, significant attention to it has only been paid recently by researchers and practitioners, with the END actions on creating quiet areas as a main driver. In the latest major international conferences including the International Congresses on Noise Control Engineering (inter-noise), Annual Conference of Association of European Schools of Planning (AESOP), International Congress on Acoustics (ICA),
International Commission on Biological Effects of Noise (ICBEN), and the European Conference on Noise Control (euro noise), a number of special sessions have been organised. Moreover, several national research projects relating to this field are being carried out. Furthermore, there are increasing interests in practice. For example, the Great London Authority is actively promoting practical exempla soundscape projects (Kang, 2009). Similar actions are also being taken in other cities, such as Berlin, Stockholm and Antwerp (Kang and Schulte-Fortkamp, 2016). A number of research networks have been formed, for example, the UK Engineering and Physical Science Research Council funded NoiseFutures Network (http:// noisefutures.org/), which brings together 65 participants from a range of academic backgrounds and experiences alongside contributions from policy makers and consultants; the EU-COST network on Soundscape of European Cities and Landscapes (http://soundscape-cost.org/), with partner organisations from 23 COST countries and 7 outside Europe, covering a range of disciplines in science, engineering, social science, humanity and medicine; and the WUN (World-wide University Network) Environmental Acoustics Network. An ISO working group has also been formed, and in the published ISO 12913-1: 2014 Acoustics-Soundscape-Part 1: Definition and conceptual framework, soundscape is defined as the 'acoustic environment as perceived or experienced and/or understood by a person or people, in context'. Thus, 'soundscape' is different from 'acoustic environment' as it relates to perceptual constructs rather than just physical phenomena.

Soundscape research represents a paradigm shift in the field of environmental noise in that it combines physical, social and psychological approaches and considers environmental sounds as a 'resource' rather than 'waste'. While many problems in current noise-only based research and policies have been revealed, there is an urgent need for such a paradigm shift. However, the current research in soundscape is still at the stage of describing and identifying the problems and tends to be fragmented and focused on only a few special cases, e.g., subjective evaluation of soundscapes especially for residential areas. The current state of knowledge and the need for standardisation of methods and indices is illustrated in special issues of scientific journals such as Journal of the Acoustical Society of America (Schulte-Fortkamp and Kang, 2017), in the EU projects (i.e., www.fp7sonorus.eu), and in a number of recent workshops organised by the networks mentioned above (Kang, 2015).

In the movement from noise control to soundscape creation, a vital step will be the standardisation of methods to assess the soundscape quality. This will be a key driver for the three major soundscape facets (Kang, 2007; Kang, 2009) -Evaluation/understanding, design, and prediction -Which all need further developments:

(1) Soundscape evaluation/understanding: Although 
much research work has been carried out on this, there is a further need to study the soundscape perception, especially the effects of various sounds on stress levels, positively or negatively. For soundscape evaluation/understanding it would be important to use standard indices for relative comparisons between different soundscapes.

(2) Soundscape design: There is a recognised need for various techniques for the overall soundscape design, going substantially beyond the current state-of-the-art which only relates to noise control. Consideration should be made from the viewpoints of the source, sound path, and receiver, and also, the design of soundscapes should be integrated into the design of the overall sustainable environment. For such soundscape design, it is vital to use standard indices of soundscape as benchmarks.

(3) Soundscape prediction/mapping: Whilst noise mapping is being intensively carried out in the EU and according to the END, agglomerations with more than 100000 inhabitants, as well as major roads, railways, and airports, must now have noise maps, there is also an urgent need for the prediction of the overall sound environment and consequently the production of soundscape maps, considering both positive and negative sounds as perceived by users (Aletta and Kang, 2015). For this, indices for soundscape are essential. Moreover, with 3D visualization tools in urban design, it is important to integrate 3D acoustic animation, taking urban sound sources into account, and their spatial and temporal conditions.

\section{From dBA to soundscape indices}

There are three basic physical indices for sound: (1) Sound power: The rate at which acoustic energy is transferred from a vibrating source to a medium; (2) sound intensity: The average rate at which sound energy is transmitted perpendicular to a specified direction; and (3) sound pressure: Incremental change from the static pressure caused by a sound wave. The earliest and most commonly used scientific index for measuring sound is the decibel (dB). It is one tenth of a bel (B), which was devised by the Bell Telephone Laboratories to quantify the reduction in audio level over a 1-mile length of standard telephone cable. $\mathrm{dB}$ is a logarithmic unit that expresses the magnitude of a sound pressure, sound intensity or sound power relative to a reference level. $\mathrm{dB}$ is useful for evaluating sound levels because exponential changes of magnitude are perceived by humans as being linearly related, i.e., a doubling of sound intensity causes perceived intensity to increase by roughly the same amount. Its logarithmic nature allows very large or very small ratios in sound pressure (or intensity, or power) to be represented by a number conveniently perceived by humans.

With a given $\mathrm{dB}$, people perceive sound differently at different frequencies in terms of subjective loudness. Since the 1930s (Fletcher and Munson, 1933), several versions of equal-loudness-level contours have been obtained, by comparative subjective measurements in a free field involving sinusoidal tones. The unit of loudness level is phon. In many cases, loudness and annoyance are two separate and operationally distinct perceived attributes. Based on laboratory subjective tests, contours of equal noisiness have also been established (Kryter, 1970). Noisiness was initially introduced for aircraft noise evaluation, but it has also been used for other kinds of noise.

To represent the overall sound level, a single value on one scale is often desirable, for which a number of different values representing sounds at various frequencies must be combined. Several frequency weighting networks have been developed, considering a typical human response to sound based on the equal-loudness-level contours, when the sound level in each frequency is adjusted and then added. Among various weighting systems, the A-weighting, with resultant decibel values called dBA (usually in the form of Leq, equivalent continuous sound level, in time domain) has been most commonly used in almost all the national/international regulations. However, there have been numerous criticisms on its effectiveness in the last decades (Parmanen, 2007). Many studies have shown that $\mathrm{dBA}$ is not well correlated with human perception and its applicability as a noise annoyance indicator relating to regulations has been in serious doubt (Hellman and Zwicker, 1987). For example, dBA was originally designed to approximate the response of the human ear at relatively low sound levels, so that its applicability range is limited. Moreover, there are increasing complaints regarding low frequency noise problems, for which dBA cannot give correct indications, on which numerous research papers have been published in the Journal of Low Frequency Noise, for example. The problems of using dBA have led to two strands of research: On adjusted level requirements for different kinds of noise sources, such as so-called railway noise bonus; and socio-psychological noise perception models for certain noise sources (Kang, 2007). However, those works are still concentrating on noise rather than the overall environments considering both negative/unwanted and positive/wanted sounds.

Psychoacoustic research is a further development from $\mathrm{dBA}$, originated in the field of industrial products, for better designing product sound quality. In the very beginning (i.e., 1980's) it just expressed that acoustic emissions had further characteristics than just level (Blauert and Jekosch, 1997). Linked to the concept of product quality, sound quality was defined as the 'adequacy of a sound in the context of a specific technical goal and/or task'. Sound quality has three main aspects: (1) Stimulus-response compatibility, which is the functional aspect of a sound; (2) pleasantness of sounds, which is based on an instantaneous overall impression emerging from various sound attributes as well as individual preferences and experience; and (3) identifiability of 
sounds or sound sources. Psychoacoustic factors, including loudness, fluctuation strength or roughness, sharpness, and pitch strength (Zwicker and Fastl, 2007) have been proved to be successful for the assessment of sound quality. The psychoacoustic factors allow for an instrumental prediction of attributes of sound perception, although instruments are still far from simulating human sound perception and evaluation in all its facets (National Instruments, 2014). More importantly, while for the sound quality of products only a single sound is usually considered, a significant feature of environmental acoustics is that there are multiple and dynamic sound sources and the use of psychoacoustic indices becomes much more complicated (Fastl, 2006).

Overall, there is a general consensus that for the assessment of acoustic environments it is not appropriate to rely on physical metrics alone and the human experience in context should also be taken into account, on the grounds that "human responses should not be equated to acoustic measures" (Andringa et al., 2013). Cases have been reported where local authorities were fulfilling all environmental noise requirements and community complaints were still being received. Indeed, while the conventional noise control engineering methods only aim at reducing sound levels, the soundscape approach acknowledges that the nature of sounds is also important and sounds might be 'wanted' as well as 'unwanted'. Therefore, with the inevitable tendency of moving from purely noise reduction to the soundscape, there is an urgent need for developing soundscape indices, with which sound environments can be classified and/or categorised for the purpose of planning or environmental assessment. This will make it possible to assess the quality of overall sound environments in a way which adequately reflects levels of human comfort.

\section{Designing soundscape: A system for urban open public spaces as an example}

To design a new soundscape or to investigate an existing soundscape, it is essential to use an appropriate system to describe the designable aspects. Taking urban open public spaces as an example, a system has been proposed, as shown in Fig. 1 (Kang, 2011), where four facets are considered, namely characteristics of each sound, acoustic effects of the space, social/demographic aspect of the users, and other physical/environmental conditions.

In terms of the characteristics of each sound, the sound pressure level (SPL), spectrum, temporal conditions, source location, source movement, and the psychological and social characteristics should be considered:

(1) For sound level, both the steady-state SPL and statistical SPL should be taken into account.

(2) For frequency, if tonal components are notable, it would be useful to consider a narrowband spectrum. Any distinguished low-frequency components should also be noted, as their effects have been paid great attention recently.

(3) For temporal conditions, when the temporal pattern of a sound is systematically varied, the sound which has the high-level portion at the beginning is perceived as being louder, which might be caused by the overshoot at the onset of the sound. Moreover, the temporal conditions description should also include rate and pattern of the sound occurrence, sound sequences, and passage of time such as acoustic actions of starting/stopping, adding/ subtracting and expanding/contracting. Furthermore, the perception of a sound also varies according to its duration. The shorter the duration is, the sharper the sound is judged. Impulsive characteristics, including peak level as well as rise and fall time, should be taken into account too.

(4) The location and movement of sound sources are of particular importance. People have a natural ability to isolate sounds in relation to their approximate positions. The auditory system is also capable of detecting from a variety of acoustic events detailed information about the distance of the sound source, its velocity, the direction of its movement, and even its size and weight.

(5) Another aspect of a sound is its psychological and social characteristics. Sound figures can be natural in occurrence or selected by the will of the listener. In describing the psychological and social characteristics of a sound, it is necessary to distinguish natural and humanmade sounds, to indicate the relationship of a sound to the activities, and to identify whether a sound is related to the soundmark of a place.

In terms of the sound fields and acoustic effects of a space, relevant factors include the shape of the space, boundary materials, street/square furniture and landscape elements. In addition to reverberation, reflection pattern and/or echogram, and possible acoustic defects such as echoes and focus effects should be checked for. The general background sound and any special sound sources around a space in question should also be considered, because, for example, when one is moving from a quiet environment to a relatively loud space, the sound evaluation would be different from that when moving from a loud space to a quiet one.

In terms of the social/demographic aspects of the users, considerations should be given to factors including gender, age, place of living (i.e., local resident or from other cities), as well as their cultural and education background. The acoustic experience of the users is also important, so is the acoustic environment at their home and working places.

In terms of other aspects of physical/environmental conditions, temperature, humidity, wind, sun, luminous, and glare should be considered, as well as the visual environment and landscape and architectural features, due to their strong interactions with perceived sound environments (Kang, 2007). 


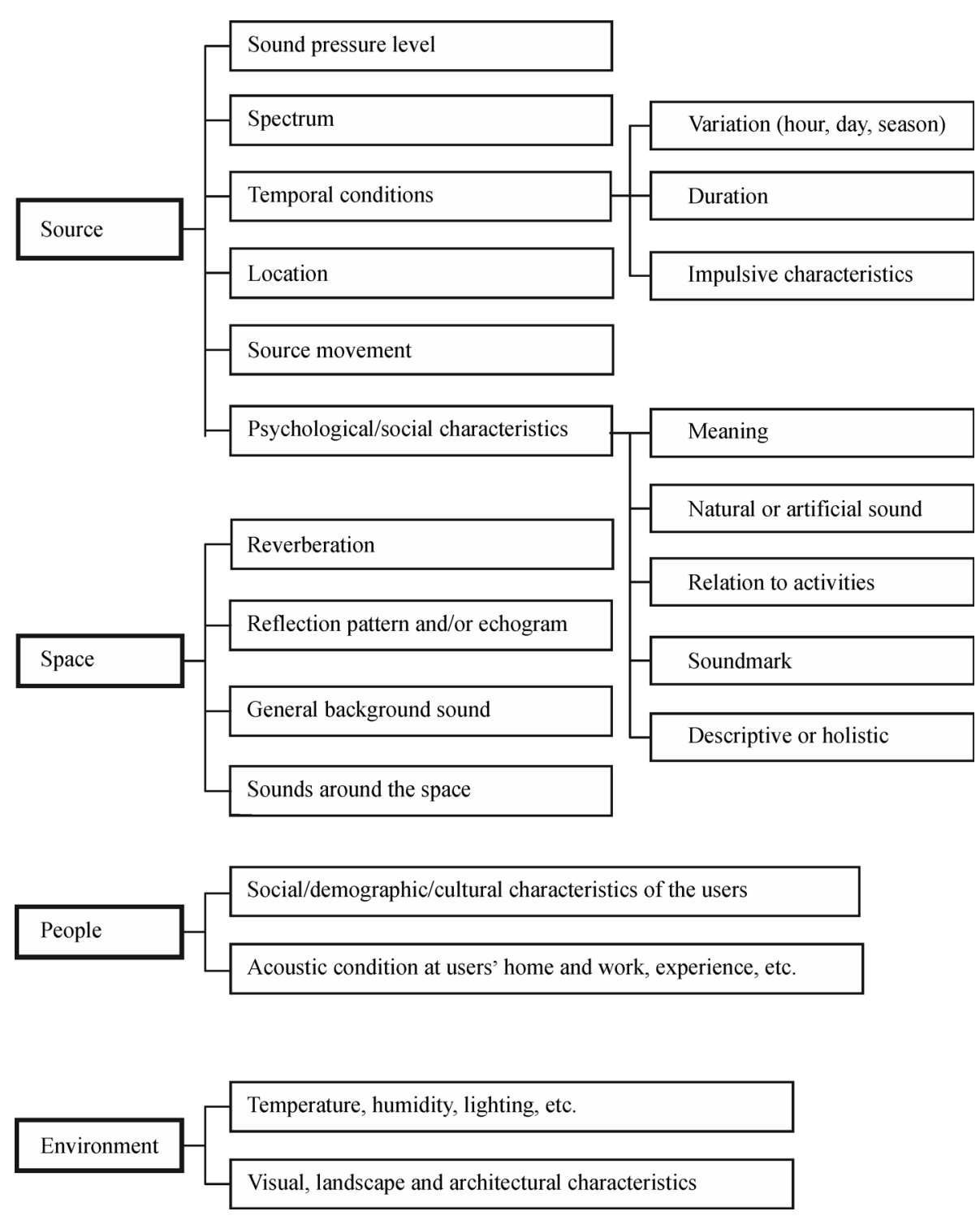

Fig. 1 A system to describe the designable aspects in urban open public spaces (Kang, 2007)

\section{A case study: Soundscapes of the water- scapes on the Gold Route in Sheffield}

A typical case is the soundscapes of waterscapes at a series of squares on the Gold Route in Sheffield, UK, as shown in Fig. 2 (Kang and Hao, 2011; Kang, 2012; Kang and Schulte-Fortkamp, 2016). This was part of an overall improvement of the gateway to the city from the main railway station, including visual improvements and enhanced environments for citizens. It includes a range of innovative water features, each with different sound characteristics, designed to attract interest and evoke city heritage. This series illustrates the powerful role of soundscapes in shaping cultural heritage and the importance of their inclusion in the regeneration of urban centres. Indeed, water has been directly related to the city's successful development, including the core role of water in the development of the market town in the 12th century, the steel industry in the 14th century, and the industrial revolution in the 19th century through to modern times.

A comprehensive study was carried out, including analysing the characteristics of different types of water features, examining the psychoacoustic factors, and field questionnaire surveys on people's perception of water soundscapes. A series of spectral analysis, from measurements taken at $1 \mathrm{~m}$ from source, showed a significant temporal and frequency variations across a variety of waterscapes along the Gold Route. The richness and diversity of the waterscapes was also demonstrated through the analysis of measurements taken when moving away from the water features, as shown in Fig. 3, where the spectral and dynamic ranges of the various soundscapes were considerable within a relatively short distance (i.e., 
$20 \mathrm{~m}$ ) from the source and the design opportunities afforded by these changes were demonstrated.

The water features in Sheaf Square provide a concert of activity creating multitudinous soundscapes varying considerably in spectral and dynamic range. A particularly interesting soundscape element was the steel barrier-It was erected to reduce noise from the adjacent road, and also to provide preferred water sounds with running water on it. The role of water in attracting attention is also important. As well as the various sounds of water, its visual effects also played a crucial role in managing the overall soundscape as demonstrated by the virtually silent installations in Millennium Square (see Fig. 2).

The analysis of psychoacoustic factors, including loudness, roughness, sharpness and fluctuation strength, also demonstrates the diversity of the soundscapes. A series of field questionnaires showed a marked preference for the sounds of the water features in the area, highlighting their noticeability: An interesting finding considering they were not the loudest sounds.

Overall this soundscape case study demonstrates the importance of utilising a diversity of sound elements (i.e., waterscapes in this case) in order to create spaces of high cultural value to enhance visitors' enjoyment of an area and reduce noise annoyance. This again demonstrates additional values the soundscape approach could provide, which would not have been achievable through a mere noise control strategy.

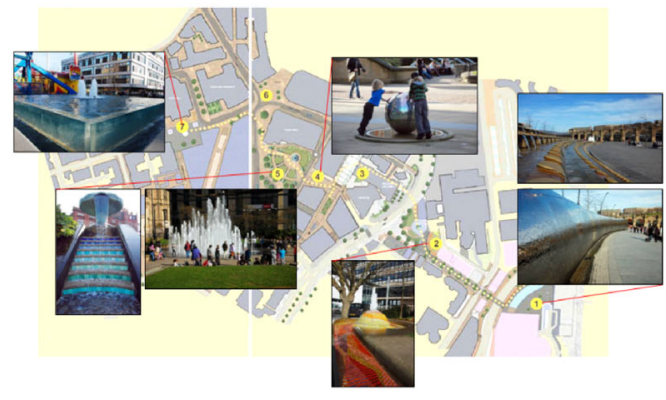

(a)

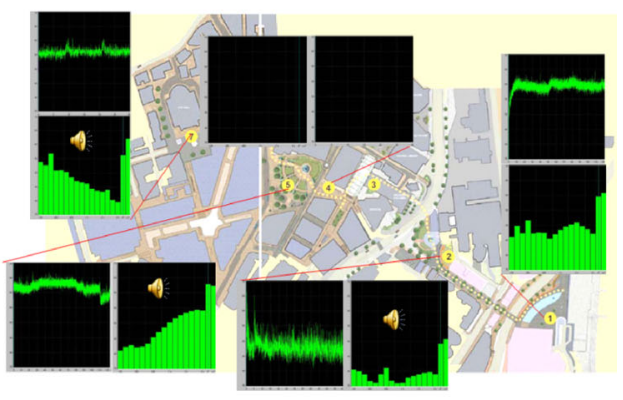

(b)

Fig. 2 The Gold Route in Sheffield

(a) The waterscape and the city; (b) changes of waterscape sound levels with frequency and time at different locations of the Gold Route, measured at $1 \mathrm{~m}$ from each water feature

1-Sheaf Square; 2-Howard Street and Hallam Garden; 3-Millennium Galleries and Winter Garden; 4-Millennium Square; 5-Peace Gardens; 6-Town Hall Square and Surrey Street; 7-Barkers Pool
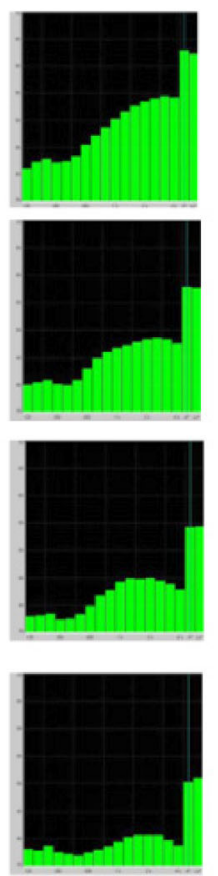

$1 \mathrm{~m}$ from source
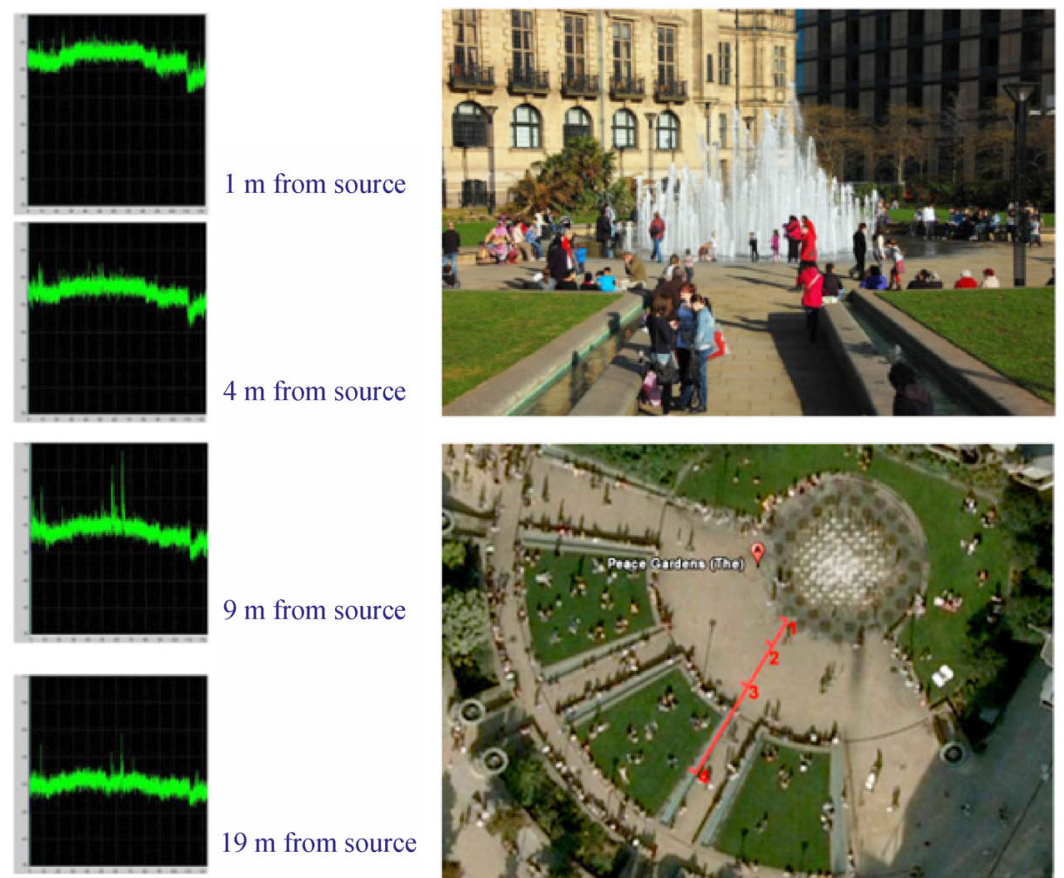

$19 \mathrm{~m}$ from source

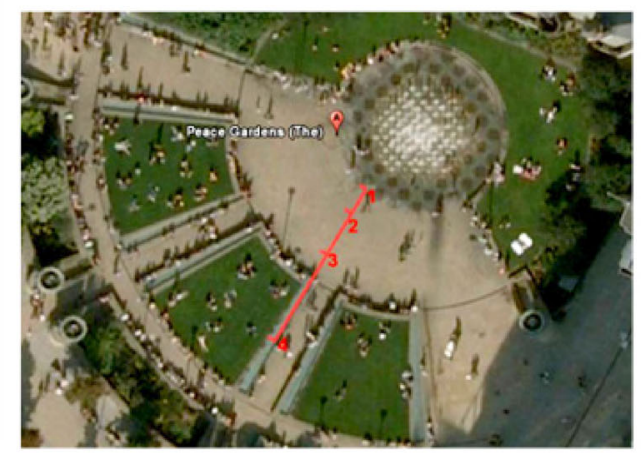

Fig. 3 Change of soundscape when moving away from the main fountain in the Peace Gardens on the Gold Route 


\section{Development and application of sounds- cape indices: A framework}

Since it has been demonstrated that people perceive/ experience different soundscapes differently (Kang, 2007; Guski, 1997; Kang, 2009), there must be fundamental factors which are determining their perceptions. Based on previous studies, they could be from three main categories, namely physiological/biological, psychological, and physical/psychoacoustical, with an additional category of contextual factors (e.g., visual, cultural) (Aletta et al., 2016).

Physiological/biological factors, which have been paid attention to recently, are proven to have considerable influence on the soundscape quality (Medvedev et al., 2015), but in this facet, relevant studies have been extremely limited. Physical/psychoacoustical factors, such as $\mathrm{L}_{\text {Aeq }}, \mathrm{L}_{90}$, loudness, sharpness, have proven to be useful but insufficient, as discussed above. Here $\mathrm{L}_{\mathrm{Aeq}}$ is A-weighted equivalent continuous sound level, and $L_{n}$ is the level of noise exceeded for $n \%$ of the specified measurement period. In other words, if $N$ measured SPL are obtained in a time period $T$ with a given time interval and they are sorted in an ascending order, then $L_{n}$ is the $(100 n / N)$ th SPL in the order. By convention, $L_{1}, L_{10}, L_{50}$ and $L_{90}$ are used to give approximate indications of the maximum, intrusive, median and background sound levels, respectively (Kang, 2007). Effects from contextual as well as psychological factors have been recognised to have an important role (Terroir et al., 2013) in the relationships between incident sound and perceived soundscape-For example, the meaning of sound sources, emergence and regularity of occasional events, number and duration of quiet periods, the amount of greenery that can be visually perceived (Watts et al., 2013), the presence of 'sound marks' and their historical value (e.g. the 'area of clear audibility of Big Ben' has been proposed for Central London), as well as users' social, demographical and behavioural factors and their expectations (Yu and Kang, 2008).

Work on the integrated effects of the above factors has been rather limited. One approach is to develop some more complex factors, such as 'Slope', which is related to how often events reach the perceiving ear and to how they emerge from background noise (Memoli et al., 2008). It is noted that perceptual factors could be considered to offer the possibility for a numerical (i.e., quantitative) or a logical (i.e., 'yes/no') assessment. Another approach is to develop intermediate indices towards the overall soundscape quality, namely evaluating one aspect of soundscape quality such as eventfulness, vibrancy, tranquillity, and pleasantness, based on psychoacoustical and psychological factors, for example (Axelsson et al., 2010).

While the feasibility of deriving soundscape indices is underlined by the fact that there are tendencies/correlations between soundscape quality and physiological/biological, psychological, physical/psychoacoustical, and contextual factors, it is noted that with multiple determining factors the establishment of soundscape indices might be complicated. Although some small-scale studies have been carried out, especially in physical/psychoacoustical and psychological facets, the results are not comparable among them and thus cannot be used directly for integrated investigations for deriving soundscape indices. To derive soundscape indices, a large-scale, interdisciplinary, nonconventional, coherent and systematic approach is important.

To address this need, it is vital to identify the determining factors of soundscape quality, shed light on how these factors affect soundscape qualities, and propose credible soundscape indices with broad application. A model framework is shown in Fig. 4, where it can be seen that the soundscape indices may take a form of a single index or a set of indices. The former could be SSID = $f$ (physical factors $)+f$ (psychological factors $)+f$ (physiological factors $)+f$ (contextual factors)..., where SSID could be a single numerical indicator, or a fuzzy indicator of possibilities. The SSID could also be calculated with a computer model, rather than the above analytical/empirical formula if there are multiple/complicated correlations among the determining factor. If it is the latter, the SSID will be based on a set of formulas or computer models, reflecting multiple attributes, for example, subjective loudness, sound preference, vibrancy, etc. Either for a single index or a set of indices, two situations should be considered, for the design stage and for the post-occupancy evaluation stage. For the former, all the input should be available at the design stage, although perceptual-related factors could be introduced based on the data of previous/ existing surveys.

In the framework of developing soundscape indices, which addresses wider intellectual goals of moving from noise reduction to soundscape creation, and make it possible to assess the quality of sound environments in a

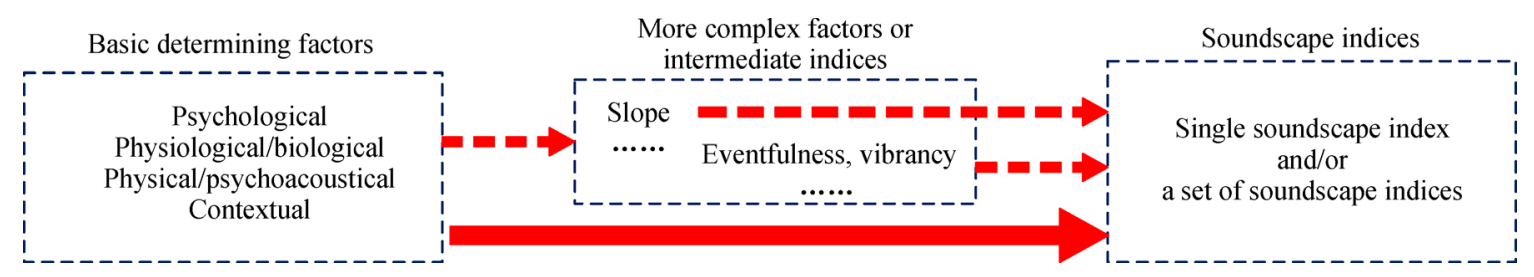

Fig. 4 Model framework of soundscape indices 
way that adequately reflects levels of human comfort, fundamental questions to be addressed include:

(1) What are soundscape characteristics and in what context are soundscape indices needed?

(2) What are the determining factors (i.e., psychological, physical/psychoacoustic, and physiological/biological), for soundscape quality and how do they affect soundscape quality?

(3) What are the routes to deriving soundscape indices?

(4) In what frameworks can the soundscape indices be applied, considering soundscape prediction, design, and standardisation?

\section{Significance and potential impact}

The development of soundscape indices will be a major step forward. This should replace dBA which has been used in sound-related regulations and standards for decades. The cost of continuing with dBA systems, which is not well related to the quality of life, will be significant, given that, for example, the annual UK cost of noise impacts is 7 billion-10 billion GBP (defra.gov.uk), and in western Europe 1 million healthy life years are lost every year from traffic-related noise (who.int/; eea.europa. eu).

Such a development would significantly enhance underpinning science for soundscape, by fostering interdisciplinary cross breeding of emerging scientific ideas related to architecture, planning, landscape, acoustics, engineering, psychology, sociology, physiology, biology, auditory perception, and cognition. The integration of medical/bioacoustic/physiological methods into the soundscape research will make the field go substantially beyond the current state-of-the-art (Brown et al., 2011; Kang et al., 2016).

The SSID will support the implementation of soundscapes - By integrating in planning policies to better inform the management of the acoustic environments, allowing for better tailored improvements to design of the built environment compared with the existing dBA-based designs, contributing to creating a more enjoyable and liveable environment, with respect to the planning of new living and recreational areas as well as to the reshaping of unsustainable older developments. The benefits of implementing soundscapes include:

(1) Health: Research indicates that quiet areas and restorative soundscapes can benefit mental health. With the increasing numbers of elderly people in Europe, there is a need to provide supportive environments which prevent the degradation of functional health. The design/re-design of well-perceived soundscapes is also a prerequisite of an adequate and healthy learning environment for children.

(2) Economy: Attractive soundscapes can enhance property prices, create an attractive setting for economic investment or offset health costs through the provision of restorative urban spaces.

(3) Culture: Soundscape is a significant indicator in the 'sensing of places' and by considering different people's perception/evaluation rather than just noise level of a place, it supports cultural diversity in the fabric of our cities; the quality of environmental sound helps people identify with a place as being unique. Soundscape studies will also help the understanding of acoustic conservation and restoration.

\section{Conclusions}

Through reviewing the movement from noise control to soundscape creation, it is clear that there are recognised needs of changing from dBA-type of measures to soundscape indices. This is also essential for the design of soundscape, as demonstrated through examining a system for urban open public spaces and analysing typical soundscape design examples.

A framework for the development and application of soundscape indices has been proposed, where suggested coherent steps for achieving this are: Characterising soundscapes by capturing soundscapes and establishing a comprehensive database; determining key factors and their influence on soundscape quality based on the database; developing, testing and validating soundscape indices; and demonstrating the applicability of the soundscape indices in the management of our sound environment.

\section{References}

Axelsson Ö, Nilsson M, Berglund B (2010). A principal components model of soundscape perception. Journal of the Acoustical Society of America, 128(5): 2836-2846

Andringa T C, Weber M, Payne S R, Krijnders J D D, Dixon M N, Linden R, de Kock E G L, Lanser J J L (2013). Positioning soundscape research and management. Journal of the Acoustical Society of America, 134(4): 2739-2747

Aletta F, Kang J (2015). Soundscape approach integrating noise mapping techniques: A case study in Brighton, UK. Noise Mapping, 2(1): $1-12$

Aletta F, Kang J, Axelsson Ö (2016). Soundscape descriptors and a conceptual framework for developing predictive soundscape models. Landscape and Urban Planning, 149: 65-74

Blauert J, Jekosch U (1997). Sound-quality evaluation-A multi-layered problem. Acta Acustica United with Acustica, 83(5): 747-753

Berglund B, Lindvall T, Schwela D H (1999). Guidelines for Community Noise. WHO Report

Brown A L, Kang J, Gjestland T (2011). Towards standardization in soundscape preference assessment. Applied Acoustics, 72(6): 387392

Fletcher H, Munson W A (1933). Loudness, its definition, measurement and calculation. Journal of the Acoustical Society of America, 5(2): 
$82-108$

Fastl H (2006). Psychoacoustic basis of sound quality evaluation and sound engineering. In: Proceedings of the 13th International Congress on Sound and Vibration. Vienna, 324-345

Guski R (1997). Psychological methods for evaluating sound quality and assessing acoustic information. Acta Acustica United with Acustica, 83(5): 765-774

Hellman R, Zwicker E (1987). Why can a decrease in $\mathrm{dB}(\mathrm{A})$ produce an increase in loudness? The Journal of the Acoustical Society of America, 82(5): 1700-1705

Kryter K D (1970). The Effects of Noise on Man. New York: Academic Press

Kang J (2007). Urban Sound Environment. London: Taylor \& Francis Incorporating Spon

Kang J (2009). Understanding and improving soundscape quality. In: Proceedings of TRANQUIL SPACES-From Understanding Perceptions to Practical Protection. London

Kang J (2011). Noise management—Soundscape approach. In: Nriagu J O, ed. Encyclopedia of Environmental Health. Burlington: Elsevier, 174-184

Kang J, Hao Y (2011). Waterscape and soundscape in Sheffield. In: Proceedings of Meeting of the COST Action TD0804 on Soundscape Examples in Community Context. Brighton

Kang J (2012). On the diversity of urban waterscape. In: Proceedings of the Acoustics 2012 (Joint Meeting of the French Acoustical Society and UK Institute of Acoustics). Nantes

Kang J (2015). Designing and planning soundscape. In: Proceedings of 3rd International Conference on Management Technology for Environmental Noise and Vibration Control. Beijing

Kang J, Aletta F, Gjestland T T, Brown L A, Botteldooren D, SchulteFortkamp B, Lercher P, van Kamp I, Genuit K, Fiebig A, Bento Coelho J L, Maffei L, Lavia L (2016). Ten questions on the soundscapes of the built environment. Building and Environment,
108: 284-294

Kang J, Schulte-Fortkamp B (2016). Soundscape and the Built Environment. London: Taylor \& Francis Incorporating Spon

Memoli G, Licitra G, Cerchiai M, Nolli M, Palazzuoli D (2008). Measuring soundscape improvement in urban quiet areas. In: Proceedings of UK IOA Conference. Reading

Medvedev O, Shepherd D, Hautus M (2015). The restorative potential of soundscapes: A physiological investigation. Applied Acoustics, 96: $20-26$

National Instruments (2014). Measurement of Sound Quality. http:// www.ni.com/white-paper/1256/en/, 2016-12-15

Parmanen J (2007). A-weighted sound pressure level as a loudness/ annoyance indicator for environmental sounds - Could it be improved? Applied Acoustics, 68(1): 58-70

Schulte-Fortkamp B, Kang J, eds. (2017). Special issue on 'Soundscape and its Applications'. The Journal of the Acoustical Society of America, 141(6)

Terroir J, De Coensel B, Botteldooren D, Lavandier C (2013). Activity interference caused by traffic noise: Experimental determination and modeling of the number of noticed sound events. Acta Acustica United with Acustica, 99(3): 389-398

Watts G, Miah A, Pheasant R (2013). Tranquillity and soundscapes in urban green-spaces-Predicted and actual assessments from a questionnaire survey. Environment and Planning B: Urban Analytics and City Science, 40(1): 170-181

Wilhelmsson M (2010). The impact of traffic noise on the values of single-family houses. Journal of Environmental Planning and Management, 43: 799-815

Yu L, Kang J (2008). Effects of social, demographical and behavioral factors on the sound level evaluation in urban open spaces. Journal of the Acoustical Society of America, 123(2): 772-783

Zwicker E, Fastl H (2007). Psychoacoustics - Facts and Models. Berlin: Springer 